\title{
Original
}

\section{Prevalencia de sintomas del tracto urinario inferior relacionados con la hiperplasia benigna de próstata. Estudio de 1804 hombres de 40 años o más residentes en Madrid durante 1999-2000}

\author{
Cristina Fernández Pérez*, Jesús Moreno Sierra**, Sara Cano Escudero*, \\ Manuel Enrique Fuentes Ferrer*, Gloria Bocardo Fajardo**, Ángel Silmi Moyano*** \\ *Unidad de Apoyo a la Investigación. Servicio de Medicina Preventiva. Hospital Clínico San Carlos. Madrid. \\ **Servicio de Urología. Hospital Clínico San Carlos. Madrid. ***Universidad Complutense de Madrid.
}

Proyecto financiado por la Sociedad Urológica Madrileña (SUM)

\begin{abstract}
Resumen
Introducción: Los síntomas del tracto urinario inferior (STUI) asociados con la hiperplasia benigna de próstata están presentes en el 20-30\% de la población española masculina de 50 años o más. El objetivo de este estudio es analizar las características clínicas y epidemiológicas de los pacientes con STUI.

Material y métodos: Estudio transversal realizado entre los años 1999 y 2000 en 1804 hombres de 40 años o más residentes en Madrid. Mediante entrevista telefónica se recabó información sociodemográfica de los participantes y se evaluó la presencia de STUI mediante el I-PSS (International Prostate Symptoms Score). La asociación entre variables cualitativas se evaluó con el test $\chi^{2}$ o el exacto de Fisher. Se ajustó un modelo de regresión logística para evaluar la probabilidad de tener STUI moderados/severos controlando la confusión.

Resultados: La prevalencia de STUI moderados/severos fue del 16,6\% (IC95\%: 14,8-18,3). Cerca del 90\% de los participantes consumían aceite de oliva, el $71,5 \%$ alcohol, el $63,1 \%$ no fumaba y el $96,9 \%$ no consumían drogas. Un $27,7 \%$ refería hipertensión y un 8,8\% diabetes. Los hombres de 70 años o más tenían una frecuencia de STUI moderados/severos tres veces mayor que los hombres más jóvenes (OR: 3,31; IC95\%: 2,10-5,22). El nivel bajo de estudios, frente al alto, mostró una frecuencia 2,2 veces mayor de STUI moderados/severos (IC95\%: 1,42-3,46), así como el consumo de aceite de semillas, frente al de oliva (OR: 1,86; IC95\%: 0,98-3,55).

Conclusiones: La historia familiar de enfermedades urológicas, la edad, el nivel bajo de estudios, la hipertensión, la diabetes y el consumo de aceite de semillas se asociaron de forma independiente con la presencia de STUI más severos, mientras el consumo moderado de alcohol y tabaco se asoció de forma independiente a la presencia de STUI leves.
\end{abstract}

Palabras Clave: Epidemiología. Tracto urinario. Signos y síntomas. Prevalencia. Hiperplasia de próstata.

\section{Prevalence of lower urinary tract symptoms related with benign prostatic hyperplasia.} Study of 1804 men aged 40 or older in Madrid

\section{Abstract}

Introduction: Lower urinary tract symptoms (LUTS) related with benign prostatic hyperplasia (BPH) are present nowadays in approximately 20 to $30 \%$ of the Spanish male population from the age of 50 onwards. The purpose of this study was to assess clinical and epidemiological characteristics of patients with LUTS.

Methods: A cross-sectional study was performed, between 1999 and 2000, among 1,804 men aged 40 or older who were living in Madrid. Subjects were interviewed by telephone; socio-demographic information was requested and the presence of LUTS was assessed using the International Prostate Symptoms Score. Informed consent was requested. Association between qualitative variables was evaluated by $\chi^{2}$ or Fisher's test. A logistic regression model was performed to control confusion.

Results: Prevalence of moderate/severe LUTS was 16.6\% (95\%CI: 14.8-18.3). Nearly 90\% of the subjects consumed olive oil, $71.5 \%$ alcohol, $63.1 \%$ did not smoke and $96.9 \%$ did not consume drugs. A $27.7 \%$ of the subjects had hypertension and $8.8 \%$ referred diabetes. Men aged 70 or older had a threefold increased frequency of serious symptoms compared to younger men (OR: 3.31; 95\%CI: 2.10-5.22). Low level of studies increased this frequency by a factor of 2.2 (95\%CI: 1.42-3.46) and men who consumed only seed oil had twice more serious symptoms than those who consumed olive oil (OR: 1.86; 95\%CI: 0.98-3.55).

Conclusions: Family history of urological diseases, age, low level of studies, hypertension, diabetes and seed oil consumption were independently associated with more serious symptoms, while medium alcohol consumption and mild smoking habit were associated with slighter symptoms.

Keywords: Epidemiology. Urinary tract. Signs and symptoms. Prevalence. Prostatic hyperplasia. 
$\mathrm{E}^{n}$ la actualidad el 20-30\% de los hombres, a partir de la quinta década de la vida, presenta Síntomas del Tracto Urinario Inferior (STUI) relacionados con la Hiperplasia Benigna de Próstata (HBP $)^{1-5}$, coincidiendo con la situación actual en Europa ${ }^{1,6,7}$. Diversos estudios muestran que la prevalencia de STUI se incrementa con la edad ${ }^{8}$ y algunos autores sugieren que aproximadamente el 43\% de los hombres de más de 60 años tiene alterada su calidad de vida debido a la presencia de síntomas urinarios $^{7,9,10}$. España cuenta en la actualidad con más de 39 millones de habitantes, de los cuales cerca de 19 millones son hombres (5,2 millones mayores de 50 años y 1,4 millones mayores de 70 años), lo que conduce a un número importante de sujetos subsidiarios de atención urológica. Esta situación podría explicar el aumento del gasto sanitario por STUI y HBP en nuestro país en los últimos años. Por otro lado, debido al envejecimiento de la población española, la prevalencia de STUI asociados a HBP se espera que aumente, así como el incremento en la expectativa de vida, lo que implica un aumento en el total de los costes relacionados con los tratamientos ${ }^{11}$.

El objetivo principal de este trabajo es analizar las características clínicas y epidemiológicas de los individuos con STUI asociados a HBP, siguiendo las recomendaciones de la Organización Mundial de la Salud.

\section{MÉTODOS}

\section{Diseño y sujetos de estudio}

Se realizó un estudio transversal entre los varones de 40 años o más que residían en la Comunidad de Madrid, excluyendo los individuos con historia previa de cirugía prostática, uretral o vesical, enfermedades neurológicas que afecten al vaciamiento vesical (esclerosis múltiple, traumatismo medular, tumores de médula, vejiga neurógena, complicaciones derivadas de la diabetes mellitus), enfermedades psiquiátricas, problemas socioeconómicos, neoplasias de próstata y vejiga, estenosis uretral y utilización de medicamentos que afecten a la fisiología del tracto urinario inferior $\left(\right.$ Tebetane $^{\circledR}$, Proscar $^{\circledR}$, Omnic $^{\circledast}$, Urolosin ${ }^{\circledast}$, Cardurán ${ }^{\circledast}$, Alfetin ${ }^{\circledR}$, Magnurol $^{\circledR}$, Benestán $^{\circledR}$, Pronitol ${ }^{\circledR}$, Deflox $^{\circledR}$ y Progandol ${ }^{\circledR}$.

\section{Tamaño muestral y selección de la muestra}

Se calculó un tamaño muestral en base a un nivel de confianza del 95\%, una precisión del 2,2 y una prevalencia estimada de síntomas moderados/severos del 30\% ${ }^{3}$. A continuación, se estratificó el tamaño muestral por edades, resultando necesarios un total de 1.665 individuos. La asignación muestral por estrato se realizó proporcionalmente a la población residente en cada estrato (muestreo estratificado con afijación proporcional a partir de la base de datos de tarjeta sanitaria). Como resultado del trabajo de campo, la muestra válida finalmente obtenida presentó ligeras modificaciones respecto a la asignación inicial, realizándose un total de 1804 entrevistas.

\section{Variables y herramientas}

Las entrevistas telefónicas fueron realizadas por personal adecuadamente entrenado entre el 23 de Noviembre de 1999 y el 14 de Enero de 2000. La entrevista se estructuró en dos partes, una primera con datos epidemiológicos (demográficos, nivel de estudios y ocupación, antecedentes médicos familiares y personales, nutrición y hábitos de vida), y una segunda para valorar la presencia de STUI. La herramienta epidemiológica se diseñó a partir de la Encuesta Nacional de Salud ${ }^{12}$ y la presencia de STUI se valoró mediante la versión validada al castellano del International Prostate Symptom Score (I-PSS) ${ }^{13}$. Este índice se construye mediante la suma de las puntuaciones de los 7 items y se clasifica en las siguientes categorías de severidad de STUI: asintomáticos (I-PSS $=0$ ), síntomas leves (I-PSS=1-7), moderados (I-PSS=8-19) y severos (I-PSS $=20-35)$.

\section{Ética}

Se solicitó el consentimiento informado verbal previa cumplimentación de la encuesta, respetando las normas de la declaración de Helsinki. La confidencialidad de los datos se mantuvo de acuerdo a la ley de protección de datos. El protocolo fue aprobado por el Comité de Ética e Investigación Clínica del Hospital Clínico San Carlos.

\section{Análisis estadístico}

Las variables cualitativas se presentan con su distribución de frecuencias junto a su intervalo de confianza al 95\%. Las variables cuantitativas se resumen en su media y desviación estándar (DE). Las variables cuantitativas que muestran una distribución asimétrica se resumen con la mediana y el rango intercuartílico (RIC: $\mathrm{P}_{25}-\mathrm{P}_{75}$ ). La asociación 
entre variables cualitativas se evaluó con el test de $\chi^{2}$, o con la prueba exacta de Fisher en el caso de que más de un 25\% de los esperados fueran menores de 5. En el caso de variables ordinales se contrastó la hipótesis de tendencia ordinal de proporciones. Se estimó la “odds ratio" (OR) junto a su intervalo de confianza al 95\% según el método de Cornfield.

Se ajustó un modelo de regresión logística, incluyendo aquellas variables que en el análisis crudo mostraron una p de contraste inferior a 0,15 y aquellas con relevancia clínica. En todos los contrastes de hipótesis se rechazó la hipótesis nula con un error de tipo I menor al 5\% $(\alpha<0,05)$. Como herramientas para realizar el análisis estadístico se utilizaron el paquete informático SPSS 12.0, EPIDAT ver 2.0 y EPIINFO ver 6.0 .

\section{RESULTADOS}

La prevalencia de STUI moderados/severos fue de $16,6 \%$ (IC95\% 14,8-18,3). El 93,3\% de los participantes provenía de un hábitat urbano (93,3\%), el 89,0\% estaban casados, el 57,4\% refería un nivel intermedio de estudios, el $34,1 \%$ tenían entre 40 y 49 años y el 62,5\% eran trabajadores en el momento del estudio (Tabla 1).

La media de peso fue de $76,2 \mathrm{Kg}(\mathrm{DE}: 11,3)$ y la de altura 170,3 cm (DE:7,1). Casi el 90\% de los sujetos consumían aceite de oliva, un $71,5 \%$ alcohol, un $63,1 \%$ no fumaban $\mathrm{y}$ un $96,9 \%$ no consumían drogas (Tabla 2).

Un $21,5 \%$ de los sujetos presentaron historia familiar de enfermedad prostática y un 6,2\% de cáncer. Hasta un 11\% de los sujetos se habían sometido a vasectomía. Un $27,7 \%$ de los sujetos refirieron hipertensión (HTA), de los cuales un $16,7 \%$ estaba en tratamiento. Un $8,8 \%$ de los sujetos presen-

taban diabetes mellitus, de los cuales un 3,6\% estaban en tratamiento oral y un 1,0\% en tratamiento con insulina (Tabla 3).

Se realizó un análisis univariado para estimar la probabilidad de tener síntomas moderados/severos (IPSS $\geq 8$ ) frente a sintomas leves o no tenerlos.

Como se muestra en la Tabla 4, la frecuencia de sintomas más severos tiende a incrementarse con la edad $\mathrm{y}$, específicamente, la probabilidad de tener IPSS $\geq 8$ en hombres de más de 70 años era 4,5 veces mayor que la de los hombres de entre 40 y 49 años. Los individuos con bajo nivel de estudios resultaron tener 3,1 veces más frecuencia de síntomas moderados/severos que los sujetos con estu-

Tabla 1. Características sociodemográficas de la población estudiada ( $\mathrm{N}=1804)$.

The
$\%$ (n)

HÁBITAT
Urbano
Rural

\section{ESTADO CIVIL}

Soltero

Casado

Viudo

Separado o divorciado

NIVEL DE ESTUDIOS

Bajo

Medio

Alto

\section{CATEGORÍA PROFESIONAL}

Fuerzas armadas

Dirección de empresas y administración pública

Técnicos y profesionales científicos

Técnicos y profesionales de apoyo

Empleados de tipo administrativo

Trabajadores de servicios

Trabajadores cualificados en agricultura y pesca

Artesanos y trabajadores cualificados

Operadores de instalaciones, maquinaria y montadores

Trabajadores no cualificados

No ha trabajado nunca

No contesta

SITUACIÓN LABORAL ACTUAL
Activo
Parado
Jubilado

EDAD (años)
$40-49$
$50-59$
$60-69$
$\geq 70$

57,4 (1035)

$22,6(408)$

$93,3(1683) \quad 92,0-94,4$

$6,7(121) \quad 5,6-7,9$

$\begin{array}{cc}5,0(90) & 4,0-6,1 \\ 89,0(1606) & 87,5-90,4 \\ 3,7(66) & 2,8-4,6 \\ 2,3(42) & 1,7-3,1\end{array}$

$18,2-21,9$

$55,1-59,7$

$20,7-24,6$

$\begin{array}{cc}1,5(27) & 1,0-2,2 \\ 11,1(201) & 9,8-12,7 \\ 12,6(227) & 11,1-14,2 \\ 10,3(185) & 8,9-11,7 \\ 10,1(182) & 8,8-11,6 \\ 8,5(153) & 7,3-9,9 \\ 1,5(27) & 1,0-2,2 \\ 22,7(410) & 20,9-24,7 \\ 13,6(245) & 12,1-15,2 \\ 7,4(133) & 6,3-8,7 \\ 0,7(12) & 0,4-1,2 \\ 0,1(2) & 0,0-1,4\end{array}$

$62,5(1127)$

$3,7(67)$

$60,2-64,7$

$33,8(610)$

$2,9-4,7$

$31,6-36,1$

$56,5(11,32)^{*}$

$34,1(615) \quad 31,9-36,3$

$28,3(511) \quad 26,3-30,5$

$22,4(404) \quad 20,5-24,4$

$15,2(274) \quad 13,6-16,9$

* Media (desviación estándar);IC: intervalo de confianza 
Tabla 2. Características constitucionales y hábitos de vida de la población estudiada $(\mathrm{N}=1804)$.

\begin{tabular}{|c|c|c|}
\hline & $\begin{array}{c}\text { Frecuencia } \\
\% \text { (n) }\end{array}$ & IC $95 \%$ \\
\hline PESO (kg) & $76,2(11,3)^{*}$ & \\
\hline TALLA (cm)a & $170,3(7,1)^{*}$ & \\
\hline $\mathrm{IMC}\left(\mathrm{kg} / \mathrm{m}^{2}\right)$ & $26,3(3,4)^{*}$ & \\
\hline Normal $(\leq 25)$ & $37,9(675)$ & $35,6-40,2$ \\
\hline Sobrepeso $(25,00-29,99)$ & $49,5(881)$ & $47,1-51,8$ \\
\hline Obesidad $(\geq 30)$ & $12,6(225)$ & $11,1-14,3$ \\
\hline \multicolumn{3}{|l|}{ CONSUMO DE ACEITE } \\
\hline Oliva & $87,8(1574)$ & $86,2-89,3$ \\
\hline Semillas & $3,4(60)$ & $2,6-4,3$ \\
\hline Oliva y semillas & $8,9(159)$ & $7,6-10,3$ \\
\hline \multicolumn{3}{|l|}{ COMPLEJOS VITAMÍNICOS } \\
\hline Sí & $4,4(79)$ & $3,5-5,4$ \\
\hline No & $95,6(1724)$ & $94,6-96,5$ \\
\hline \multicolumn{3}{|l|}{ CONSUMO DE ALCOHOL } \\
\hline Sí & $71,5(1290)$ & $69,4-73,6$ \\
\hline No & $28,5(514)$ & $26,4-30,6$ \\
\hline \multicolumn{3}{|c|}{ NIVEL DE consumo de alcohol (g/día) } \\
\hline 0 & $30,0(539)$ & $27,9-32,1$ \\
\hline $0,1-25,9$ & $52,7(948)$ & $50,4-55,0$ \\
\hline $26,0-74,9$ & $15,5(278)$ & $13,9-17,2$ \\
\hline$\geq 75$ & $1,9(34)$ & $1,4-2,6$ \\
\hline \multicolumn{3}{|l|}{ CONSUMO DE TABACO } \\
\hline Sî & $36,9(666)$ & $34,7-39,2$ \\
\hline No & $63,1(1138)$ & $60,8-65,3$ \\
\hline \multicolumn{3}{|l|}{ TIPO DE FUMADOR } \\
\hline Nunca fumadores & $20,1(363)$ & $18,3-22,1$ \\
\hline Exfumadores & $43,0(775)$ & $40,7-45,3$ \\
\hline Cigarrillos & $34,2(616)$ & $32,0-36,4$ \\
\hline Puros & $2,5(45)$ & $1,8-3,3$ \\
\hline Pipas & $0,3(5)$ & $0,1-0,7$ \\
\hline \multicolumn{3}{|c|}{ CANTIDAD DE CIGARRILLOS (nº cigarrillos/día) } \\
\hline 0 & $20,8(363)$ & $19,0-22,8$ \\
\hline $1-20$ & $25,1(438)$ & $23,1-27,2$ \\
\hline $21-34$ & $5,4(94)$ & $4,4-6,6$ \\
\hline$\geq 35$ & $4,2(74)$ & $3,4-5,3$ \\
\hline \multicolumn{3}{|l|}{ CONSUMO DE DROGAS } \\
\hline Sí & $3,1(56)$ & $2,4-4,0$ \\
\hline No & $96,9(1747)$ & $96,0-97,7$ \\
\hline
\end{tabular}

* Media (desviación estándar); IC: intervalo de confianza; IMC: índice de masa corporal
Como se observa en la Tabla 6, los hombres que tenían diabetes, hipertensión o historia familiar de STUI, presentaban, respectivamente, una frecuencia de sintomas severos 2,09 (IC95\% 1,40-3,03), 1,77 (IC95\% $1,36-2,29)$ y 1,74 (IC95\% 1,32$2,31)$ veces mayor. Aunque se constató una mayor frecuencia de hombres asintomáticos entre aquellos que se habían sometido a vasectomía, estas diferencias no resultaron significativas al estratificar en función del IPSS ( $<8$ ó $\geq 8$ ).

Se observó una interacción entre el hábito de fumar y el índice de masa corporal (IMC) en relación a la sintomatología más severa; los hombres que fumaban entre 21-34 cigarrillos por día y tenían un IMC normal (IMC $\leq 25 \mathrm{Kg} / \mathrm{m}^{2}$ ) tenían menor frecuencia de síntomas moderados/severos (Fig. 1).

Se construyó un modelo de regresión logística, ajustando por edad, historia familiar de enfermedades urológicas, HTA, diabetes, tipo de aceite, alcohol, tabaco y nivel de estudios. La historia familiar de enfermedades urológicas, la historia personal de HTA y diabetes, el nivel de estudios y la edad se asociaron de manera independiente a la presencia de sintomatología moderada/severa (Fig. 2). Los hombres de 70 o más años presentaron tres veces más frecuencia de síntomas severos dios superiores. En relación al estado civil, los viudos presentaron mayor frecuencia de síntomas moderados/severos.

En cuanto a los hábitos, el consumo de alcohol, tabaco y otras drogas se asociaron de manera significativa con una menor prevalencia de síntomas moderados/severos, aunque la edad podría estar confundiendo esta asociación (Tabla 5). que los hombres por debajo de 50 años (OR: 3,31; IC95\% 2,10-5,22). La frecuencia de síntomas moderado/severos en los que tenían un nivel bajo de estudios era 2,22 veces mayor (IC95\% 1,42-3,46) que la de los hombres con estudios superiores. El tipo de aceite utilizado se asoció de forma significativa e independiente con la presencia de síntomas moderados y severos, presentando los varones que 
Tabla 3. Antecedentes familiares y personales de la población estudiada $(\mathrm{N}=1804)$.

\begin{tabular}{|c|c|c|}
\hline & $\begin{array}{c}\text { Frecuencia } \\
\text { n (\%) }\end{array}$ & IC 95\% \\
\hline \multicolumn{3}{|c|}{ ANTECEDENTES FAMILIARES PROSTÁTICOS } \\
\hline Sí & $21,5(383)$ & $19,6-23,5$ \\
\hline No & 78,5 (1399) & $76,5-80,4$ \\
\hline \multicolumn{3}{|c|}{ TIPO DE ANTECEDENTES FAMILIARES } \\
\hline Cáncer & $6,2(110)$ & $5,1-7,4$ \\
\hline Otros & $15,3(273)$ & $13,7-17,1$ \\
\hline Ninguno & 78,5 (1399) & $76,5-80,4$ \\
\hline \multicolumn{3}{|l|}{ VASECTOMÍA } \\
\hline Sí & $10,9(197)$ & $9,5-12,5$ \\
\hline No & $89,1(1606)$ & $87,5-90,5$ \\
\hline \multicolumn{3}{|l|}{ HIPERTENSIÓN ARTERIAL (HTA) } \\
\hline Sí & 27,7 (498) & $25,7-29,9$ \\
\hline No & $72,3(1298)$ & $70,1-74,3$ \\
\hline \multicolumn{3}{|l|}{ HTA Y TRATAMIENTO } \\
\hline Sí, con tratamiento & $16,7(300)$ & $15,1-18,5$ \\
\hline Sí, sin tratamiento & $11,0(198)$ & $9,6-12,6$ \\
\hline No & 72,3 (1298) & $70,1-74,3$ \\
\hline \multicolumn{3}{|l|}{ DIABETES } \\
\hline Sí & $8,8(159)$ & $7,6-10,2$ \\
\hline No & $91,2(1642)$ & $89,8-92,4$ \\
\hline \multicolumn{3}{|l|}{ DIABETES Y TRATAMIENTO } \\
\hline Sí, con insulina & $1,0(18)$ & $0,6-1,6$ \\
\hline Sí, con antidiabéticos orales & $3,6(65)$ & $2,8-4,6$ \\
\hline Sí, sin tratamiento & $4,2(76)$ & $3,3-5,3$ \\
\hline No & $91,2(1642)$ & $89,8-92,4$ \\
\hline
\end{tabular}

IC: intervalo de confianza

consumían sólo aceite de semillas 1,86 veces más síntomas que los que consumían sólo aceite de oliva (IC 95\%: 0,98-3,55).

\section{DISCUSIÓN}

La Organización Mundial de la Salud (OMS) aconseja conocer en profundidad el comportamiento de la población respecto a la demanda de asistencia urológica. Es bien conocido que hay pacientes cuya actividad diaria se ve afectada por los STUI y que sin embargo nunca demandan tratamiento. Es necesario explorar las razones que determinan esta conducta, así como conocer qué porcentaje de la población adopta este comportamiento ${ }^{14}$.

El presente estudio es una investigación pionera en España, fundamentada en otros estudios ${ }^{3,4,8,15}$ y basada en las recomendaciones de la OMS (París 1997) ${ }^{16}$. Es un estudio transversal diseñado para estimar la prevalencia de STUI, como un primer paso para conocer la distribución de los síntomas en la población. Aunque la prevalencia estimada de STUI moderados/severos es similar a la obtenida por otros estudios en Francia ${ }^{17}$ y Austria $^{18}$, la categoría de STUI leves del presente trabajo es más frecuente que la encontrada en otros estudios realizados en España $^{3,4}$, Canadá $^{19}$, Japón ${ }^{8,20}$ y en los Estados Unidos ${ }^{10}$, con prevalencias estimadas de un 25-30\% aproximadamente.

$\mathrm{El}$ incremento de la prevalencia de STUI con la edad que se ha detectado en este estudio, corrobora los resultados de otros trabajos publi$\operatorname{cados}^{3,4,8,10,14,16,17,19,21}$.

Varias investigaciones clínicas y epidemiológicas han intentado conocer la etiología de los STUI y sus factores desencadenantes, sin embargo, aún no se dispone de suficiente información para establecer conclusiones definitivas que puedan traducirse en estrategias preventivas, lo que justifica el diseño de nuevos estudios para identificar factores de riesgo ${ }^{23}$.

Pocos estudios han evaluado la influencia de los factores sociodemográficos. Haidinger et al. ${ }^{18}$ no encontraron relación entre los STUI moderados/ severos y el nivel educativo, los ingresos mensuales o el estado civil, mientras nuestro estudio ha observado prevalencias significativamente distintas en función del nivel de estudios, el estado civil y la situación laboral. Tras ajustar por otros factores sociodemográficos, el nivel bajo de estudios, con respecto a los hombres con un alto nivel educativo, fue el único factor independientemente asociado a la frecuencia de STUI moderados/severos.

La relación inversa encontrada entre el consumo de alcohol y los STUI confirma los resultados publicados con anterioridad. Platz et al. ${ }^{24}$ encontraron una reducción del efecto beneficioso del alcohol con consumos de $50 \mathrm{~g} /$ día o superiores mientras en nuestro estudio dicho efecto se mantiene incluso en consumidores de más de $75 \mathrm{~g}$ al día. 
Tabla 4. Análisis univariado de los factores sociodemográficos asociados a la prevalencia de STUI moderado y severo (I-PSS $\geq 8$ ) frente a asintomático/leve (I-PSS $<8$ ).

\begin{tabular}{|c|c|c|c|c|}
\hline & $\mathbf{n}$ & OR & IC $95 \%$ & $\mathbf{p}$ \\
\hline \multicolumn{5}{|l|}{ HÁBITAT } \\
\hline Urbano & 1683 & 1 & & \\
\hline Rural & 121 & 1,13 & $0,70-1,82$ & 0,63 \\
\hline \multicolumn{5}{|l|}{ ÁREA } \\
\hline Madrid municipio & 1186 & 1 & & \multirow{3}{*}{0,83} \\
\hline Madrid periferia & 490 & 0,96 & $0,72-1,28$ & \\
\hline Resto & 128 & 0,86 & $0,52-1,43$ & \\
\hline \multicolumn{5}{|l|}{ EDAD (años) } \\
\hline $40-49$ & 615 & 1 & & \multirow{4}{*}{$<0,001$} \\
\hline $50-59$ & 511 & 2,19 & $1,51-3,18$ & \\
\hline $60-69$ & 404 & 3,15 & $2,17-4,57$ & \\
\hline$\geq 70$ & 274 & 4,50 & $3,04-6,65$ & \\
\hline \multicolumn{5}{|l|}{ NIVEL DE ESTUDIOS } \\
\hline Alto & 408 & 1 & & \multirow{3}{*}{$<0,001$} \\
\hline Medio & 1035 & 1,54 & $1,08-2,21$ & \\
\hline Bajo & 361 & 3,12 & $2,11-4,62$ & \\
\hline \multicolumn{5}{|l|}{ ESTADO CIVIL } \\
\hline Soltero & 42 & 1 & & \multirow{4}{*}{0,02} \\
\hline Casado & 90 & 2,5 & $0,52-11,92$ & \\
\hline Viudo & 1606 & 4,05 & $0,98-16,84$ & \\
\hline Divorciado o separado & 66 & 6,39 & $1,39-29,39$ & \\
\hline \multicolumn{5}{|l|}{ SITUACIÓN LABORAL ACTUAL } \\
\hline Activo/Parado & 1194 & 1 & & \multirow[b]{2}{*}{$<0,001$} \\
\hline Jubilado & 610 & 2,11 & $1,64-2,71$ & \\
\hline \multicolumn{5}{|l|}{ CATEGORÍA PROFESIONAL } \\
\hline Fuerzas armadas & 27 & 2,71 & $1,04-7,02$ & \multirow{12}{*}{0,04} \\
\hline Dirección de empresas y administración pública & 201 & 1,69 & $0,98-2,91$ & \\
\hline Técnicos y profesionales científicos & 227 & 1 & & \\
\hline Técnicos y profesionales de apoyo & 185 & 1,21 & $0,67-2,17$ & \\
\hline Empleados de tipo administrativo & 182 & 1,01 & $0,55-1,86$ & \\
\hline Trabajadores de servicios & 153 & 1,81 & $1,02-3,21$ & \\
\hline Trabajadores cualificados en agricultura y pesca & 27 & 2,71 & $1,04-7,02$ & \\
\hline Artesanos y trabajadores cualificados & 410 & 1,56 & $0,97-2,54$ & \\
\hline Oper. de instalaciones, maquinaria y montadores & 245 & 1,84 & $1,09-3,08$ & \\
\hline Trabajadores no cualificados & 133 & 2,35 & $1,33-4,17$ & \\
\hline No ha trabajado nunca & 2 & - & & \\
\hline No contesta & 12 & 0,7 & $0,09-5,67$ & \\
\hline \multicolumn{5}{|l|}{ IMC } \\
\hline Normal & 675 & 1 & & \multirow{3}{*}{0,09} \\
\hline Sobrepeso & 881 & 1,30 & $0,99-1,72$ & \\
\hline Obesidad & 225 & 1,44 & $0,97-2,15$ & \\
\hline
\end{tabular}

IC: intervalo de confianza; IMC: índice de masa corporal; OR: Odds ratio

Aunque el grupo de consumo excesivo era pequeño, circunstancia que favorece las estimaciones no significativas (OR: 0,51, IC95\%: 0,18-1,47), puede que el tipo de alcohol consumido esté ejerciendo un efecto confusor.

Entre los fumadores de 1 a 34 cigarrillos/día la prevalencia de STUI moderados/severos era menor y, como el estudio de Roberts et al. ${ }^{25,26}$ había publicado previamente, esta asociación desaparecía a partir de 34 cigarrillos/día. En nuestro estudio, la frecuencia de STUI era mayor en grandes fumadores pero no había diferencias significativas entre exfumadores y no fumadores.

La interacción descrita entre el tabaco y el IMC en su relación con la frecuencia de STUI moderados/severos (los fumadores moderados con un IMC normal tenían una frecuencia de STUI moderados/severos menor que los no fumadores con IMC normal), ha sido previamente publicada por Seitter y Barret-Connor ${ }^{27}$; estos autores también describie- 
Tabla 5. Análisis univariado de los hábitos de vida asociados a la prevalencia de STUI moderados/severos (I-PSS $\geq 8$ ) frente a asintomáticos/leves (I-PSS<8).

\begin{tabular}{|c|c|c|c|c|}
\hline & $\mathbf{n}$ & OR & IC $95 \%$ & $\mathbf{p}$ \\
\hline \multicolumn{5}{|c|}{ CONSUMO DE ACEITE } \\
\hline Oliva & 1574 & 1 & & \multirow{3}{*}{0,03} \\
\hline Oliva y semillas & 159 & 1,45 & $0,97-2,18$ & \\
\hline Semillas & 60 & 1,94 & $1,08-3,50$ & \\
\hline \multicolumn{5}{|c|}{ CONSUMO DE VITAMINAS } \\
\hline No & 1724 & 1 & & \multirow[t]{2}{*}{0,99} \\
\hline Sí & 79 & 0,99 & $0,54-1,83$ & \\
\hline \multicolumn{5}{|c|}{ CONSUMO DE ALCOHOL } \\
\hline No & 514 & 1 & & \multirow[t]{2}{*}{0,001} \\
\hline Sí & 1290 & 0,63 & $0,49-0,82$ & \\
\hline \multicolumn{5}{|c|}{ CONSUMO DE ALCOHOL (g/día) } \\
\hline 0 & 539 & 1 & & \multirow{4}{*}{0,02} \\
\hline $0,1-25,9$ & 948 & 0,67 & $0,51-0,88$ & \\
\hline $26-74,9$ & 278 & 0,66 & $0,45-0,98$ & \\
\hline$\geq 75$ & 34 & 0,51 & $0,18-1,47$ & \\
\hline \multicolumn{5}{|c|}{ CONSUMO DE TABACO } \\
\hline No & 1138 & 1 & & \multirow[t]{2}{*}{$<0,001$} \\
\hline Sí & 666 & 0,6 & $0,45-0,78$ & \\
\hline \multicolumn{5}{|c|}{$\begin{array}{l}\text { CONSUMO DE TABACO } \\
\left.\text { ( }{ }^{\circ} \text { cigarrillos } / \text { día }\right)\end{array}$} \\
\hline Nunca fumador & 363 & 1 & & \multirow{5}{*}{$<0,001$} \\
\hline Exfumador & 775 & 1,15 & $0,83-1,59$ & \\
\hline $1-20$ & 438 & 0,67 & $0,45-0,99$ & \\
\hline $21-34$ & 94 & 0,32 & $0,13-0,76$ & \\
\hline$\geq 35$ & 74 & 1,09 & $0,57-2,07$ & \\
\hline \multicolumn{5}{|c|}{ CONSUMO DE DROGAS } \\
\hline No & 1747 & 1 & & \multirow[t]{2}{*}{0,03} \\
\hline Sí & 56 & 0,38 & $0,14-1,06$ & \\
\hline
\end{tabular}

IC: intervalo de confianza; OR: Odds ratio

ron que el riesgo de cirugía prostática aumentaba en los fumadores con sobrepeso u obesidad.

El consumo de aceite, vitaminas y drogas no se ha descrito en la literatura en profundidad. Un estudio reciente evaluó la asociación entre la dieta y los STUI $^{28}$, y encontró menor frecuencia de STUI severos en los consumidores de poca verdura, carne y mantequilla. Sin embargo, en nuestro estudio y tras ajustar por otros factores, la frecuencia de STUI severos era significativamente menor en los consumidores habituales de aceite de oliva. Este resultado podría deberse a los altos niveles de vitamina $\mathrm{E}$ presentes en el aceite de oliva y a su efecto antioxidante $^{29}$. Para investigar de forma adecuada la influencia de la dieta habría que utilizar una encuesta nutricional completa, lo que no es factible mediante entrevista telefónica.

Coincidiendo con otros autores, los STUI empeoran a medida que el IMC aumenta, pero esta aso- ciación desaparece tras ajustar por otras variables en los modelos de regresión. El hecho de que el peso fuera autodeclarado no explica la falta de asociación, puesto que la validez de esta medida ha sido demostrada ${ }^{30}$. Dahle et al. ${ }^{31}$ han cuestionado la utilidad del IMC para evaluar obesidad y han recomendado medir la obesidad abdominal mediante el indice cintura-cadera y Lee et al. ${ }^{32}$ encontraron una asociación bimodal entre la obesidad abdominal y los STUI, de forma que los STUI moderados y severos eran más frecuentes en la obesidad y el infrapeso, frente a los hombres con peso medio. En conclusión, la obesidad podría estar asociada con el crecimiento de la glándula prostática pero se necesita más evidencia para poder relacionar esto con los STUI.

Una de las principales limitaciones del estudio procede del propio diseño empleado; un estudio de prevalencia no puede distinguir entre factor de riesgo o pronóstico pero, por otra parte, este diseño per- 
Tabla 6. Análisis univariado de los antecedentes personales y familiares asociados a la prevalencia de STUI moderados/severos (I-PSS $\geq 8$ ) frente a asintomáticos/leves (I-PSS<8).

\begin{tabular}{lcccc}
\hline & $\mathbf{n}$ & OR & IC 95\% & $\mathbf{p}$ \\
\hline ANTECEDENTES FAMILIARES & & & & \\
$\quad$ No & 1399 & 1 & & \\
$\quad$ Sí & 383 & 1,74 & $1,32-2,31$ & $<0,001$ \\
VASECTOMÍA & & & & \\
$\quad$ No & 197 & 1 & & 0,07 \\
$\quad$ Sí & 1606 & 0,67 & $0,43-1,05$ & $0,0,001$ \\
HIPERTENSIÓN ARTERIAL & & & & \\
$\quad$ No & 1298 & 1 & & \\
$\quad$ Sí & 498 & 1,77 & $1,36-2,29$ & $<0,001$ \\
DIABETES & & & & \\
$\quad$ No & 1642 & 1 & & \\
$\quad$ Sí & 159 & 2,09 & $1,44-3,03$ & $<0,001$ \\
\hline
\end{tabular}

IC: intervalo de confianza; OR: Odds ratio

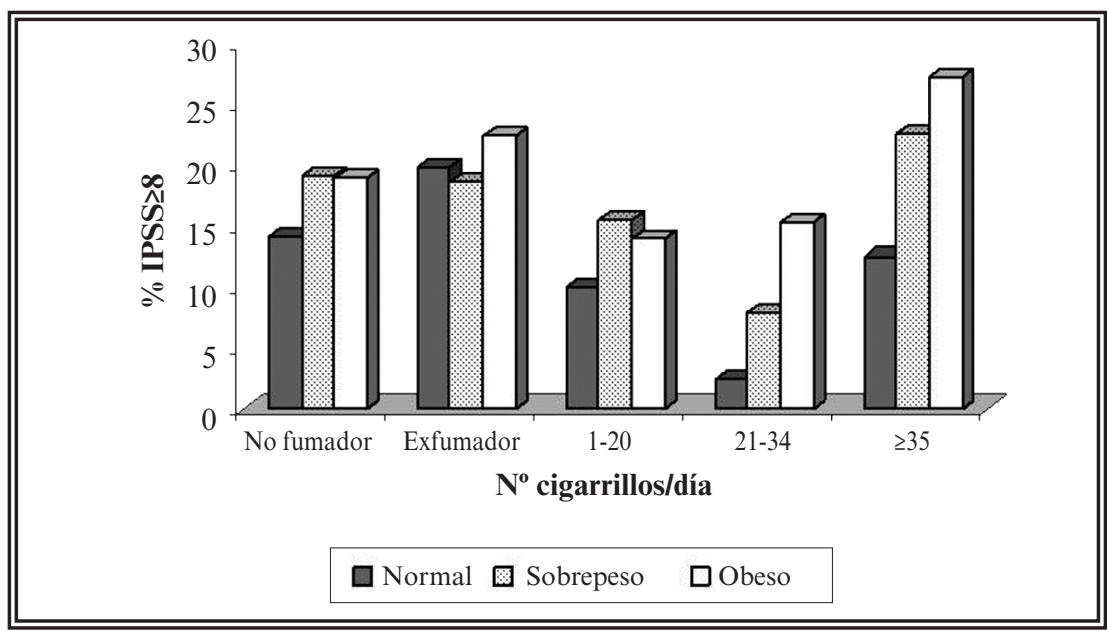

FIGURA 1. Prevalencia de sintomas moderados/severos (I-PSS $\geq 8$ ) en función de Indice de Masa Corporal y del número de cigarrillos consumidos por día.

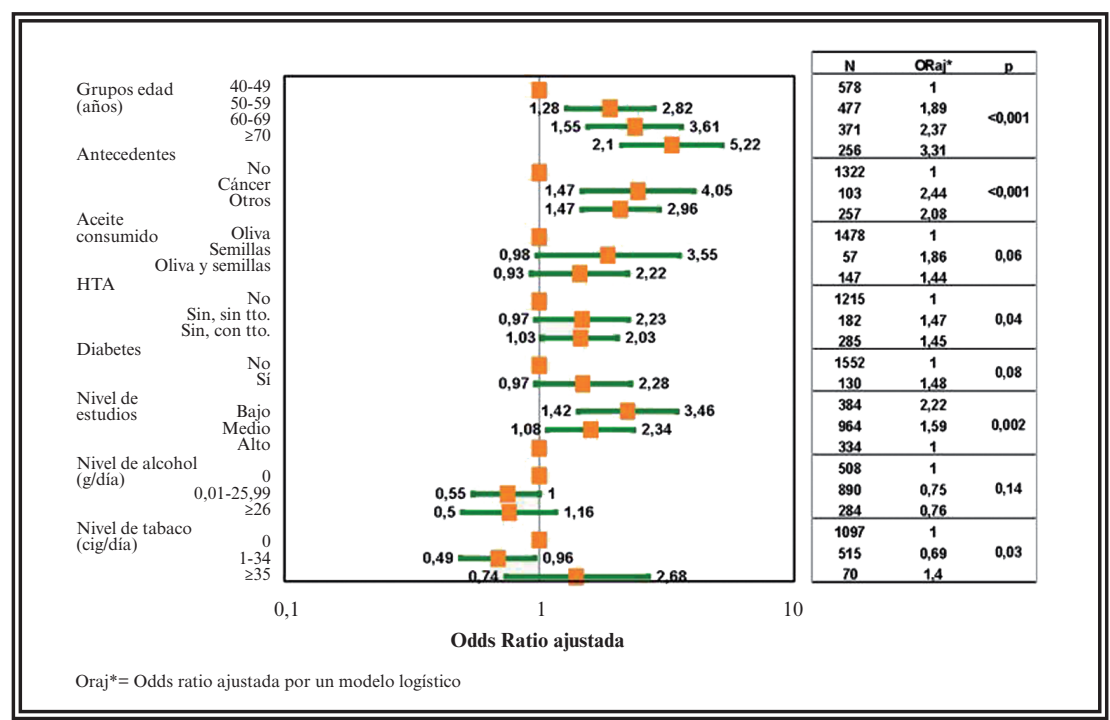

FIGURA 2. Análisis multivariado de los factores asociados a la prevalencia de síntomas moderados/severos (I-PSS $\geq 8$ ) frente a asintomático/leves (I-PSS $<8$ ). mite plantear hipótesis. La exclusión de casos prevalentes al inicio del estudio, conlleva una validez externa sólo aplicable a los hombres que no hayan sido previamente detectados por el sistema sanitario, lo que nos permite conocer las necesidades reales del área de salud.

Algunos autores han evaluado las diferencias entre las puntuaciones obtenidas en los cuestionarios en función de la técnica de recogida de la información, encontrando que las puntuaciones obtenidas mediante entrevista telefónica son menores que las del cuestionario autoadministrado $^{22}$. Por este motivo, tras finalizar la primera fase de diseño transversal, se invitó a realizar un examen urológico a los hombres cuyo I-PSS fue superior a 6 y se les administró de nuevo el cuestionario I-PSS como control de calidad del método de entrevista; se analizó la fiabilidad de ambas puntuaciones, resultando una buena concordancia entre ambas mediciones etiológicas que pueden ser evaluadas en estudios analíticos posteriores.

Finalmente, los hospitales deberían plantearse para el futuro prever la demanda de los pacientes con HBP con objeto de reducir el número de procedimientos invasivos diagnósticos y terapéuticos y así mejorar la calidad de vida de estos pacientes.

\section{CONCLUSIONES}

La prevalencia de STUI moderados/severos fue del 16,6\% en hombres de 40 años o más. La historia familiar de enfermedades urológicas, la edad, el nivel bajo de estudios, la hipertensión, la diabetes y el consumo de aceite de semillas se asociaron de forma independiente con la presencia de STUI severos, mientras el consumo moderado de alcohol y tabaco se asociaron de forma independiente con la presencia de STUI leves. 


\section{REFERENCIAS}

1. Madersbacher S, Alivizatos G, Nordling $\mathrm{J}$ et al. Guías clínicas de la EAU 2004 para la evaluación, tratamiento y seguimiento de varones con síntomas de tracto urinario inferior indicativos de obstrucción prostática benigna (Guías clínicas para la HBP) European Urology 2004;46:547-554.

2. Resel L. Etiopatogenia. Historia natural de la hipertrofia benigna de próstata. Medicine 1997;(7):9-13.

3. Chicharro Molero J, Burgos Rodríguez R, Sánchez Cruz J et al. Prevalence of benign prostatic hyperplasia in Spanish men 40 years old or older. J Urology 1998, 159(3):878-882.

4. Hunter Djw, Berra-Unamuno A, Martín-Gordo A. Prevalence of urinary symptoms and other urological conditions in Spanish men 50 years or older. J Urology 1996;155(6):1965-1970.

5. Gómez Acebo A, Rodríguez Vallejo JM, Rodríguez Mora VI et al. Calidad de vida y sintomatología en la hiperplasia prostática benigna en población activa española. Med Clin 2000;114 (Supl 3):81-89.

6. Flam T, Montauban V. Screening of clinical benign prostatic hypertrophy in general practice: survey of 18,540 men. Prog Urol. 2003 Jun. 13(3):416-424.

7. Sagnier PP, Girman CJ, Garraway M, Kumamoto Y, Lieber MM, Richard F, MacFarlane G, Guess HA, Jacobsen SJ, Tsukamoto $\mathrm{T}$, Boyle P. International comparison of the community prevalence of symptoms of prostatism in four countries. Eur Urol 1996;29:(1)15-20.

8. Tsukamoto T, Kumamoto Y, Masumori N, Miyake H, Rhodes T, Girman CJ, Guess HA, Jacobsen SJ, Lieber MM. Prevalence of prostatism in Japanese men in a community based study with comparison to similar American study. J Urology. 1995;154: 391-395.

9. Garraway W, Kirby R. Benign prostatic hyperplasia: effects on quality of life and impact on treatment decisions. Urology 1994;44(5):629-636.

10. Roehrborn CG, Marks L and Harkaway R. Enlarged prostate: a landmark national survey of its prevalence and impact on US men and their partners. Prostate Cancer Prostatic Dis 2006; 9(1):30-34.

11. Chapple CR. Lower urinary tract symptoms suggestive of benign prostatic obstruction - TRIUMPH: design and implementation. Eur Urol 2001;39 (suppl.3):31-36.

12. Ministerio de Sanidad y Consumo España. Encuesta Nacional de Salud. 1997

13. Vela Navarrete R, Martín Moreno JM, Calahorra FJ, Damián Moreno J, Hernández Coronado A, Boyle P. Validación Cultural y lengüistica en Español del International Prostatic Sympton Scale (I-PSS) Actas Urol Esp 1994;18(8):841-847.

14. Boyle P, Gu FL, Jacobsen S et al. Epidemiology and Natural History of BPH, en: Chatelain Ch, Denis L, Foo JKT, Khoury S, Connel Mc et al, eds. Proceedings of the 5th International Consultation on Benign Prostatic Hyperplasia (BPH), Paris: Jun 2000

15. Koskimäki J, Hakama M, Huhtala H et al. Prevalence of lower urinary tract symptoms in Finnish men: a population-based study. Brit J Urol 1998;81(3)64-69.

16. Oishi K, Boyle P, Barry MJ, Farah R, Gu FL, Jacobsen S et al. Epidemiology and natural history of benign prostatic hyperplasia, en: Denis L, Griffiths K, Khoury S,

17. Cockett ATK, McConell J, Chatelain C et al, Eds: Proceedings of the International Consultation on Benign Prostatic Hyperplasia, Paris: SCI, 1997;23-59.

18. Sagnier PP, MacFarlane G, Richard F, Botto H, Teillac P, Boyle P. Results of an epidemiological survey employing a modified American Urological Association Index for Benign Prostatic Hyperplasia in France. J Urology 1994;51(5):1266-270.
19. Haidinger G, Madersbacher S, Waldhoer T, Lunglmayr G, Vutuc C. The prevalence of lower urinary tract symptoms in Austrian males and associations with sociodemographic variables. Eur J Epidemiol 1999; 15(8):717-722.

20. Norman RW, Nickel JC, Fish D, Pickett SN. Prostate-related symptoms in Canadian men 50 years of age or older: prevalence and relationships among symtioms. Br J Urol 1994;74(5): 542-550.

21. Ukimura O, Kojima M, Inui E, Ochiai A, Hata Y, Watanabe M, Saitoh M, Watanabe H.. A statistical study of the American Urological Association Symptom Index for Benign Prostatic Hyperplasia in participants of mass screening program form prostatic diseases using transrectal sonography. J Urology 1996;156: 1673-1678.

22. Barry MJ, Beckley S, Boyle P, Ghoneim M, Gu FL, Guess HA et al. Importance of understanding the epidemiology and natural history of BPH, in: Cockett A, Aso Y, 23. Chatelain C, Denis L, Griffiths K, Khoury $\mathrm{S}$ et al. eds: Proceedings of the International Consultation on Benign Prostatic Hyperplasia, Paris: SCI, 1992;19-24.

24. Chatelain Ch, Denis L, Foo JKT et al. Recommendations of the International Scientific Committee: Evaluation and Treatment of Lower Urinary Tract Symptoms (LUTS) in older men. 5th International Consultation on BPH, Paris: Jun 2000.

25. White KL, Willians TF, Greenberg BG. The ecology of medical care. New Engl J Med 1961;265:885-892.

26. Platz EA, Rimm EB, Kawachi I, Colditz GA, Stampfer MJ, Willett WC, Giovannucci E. Alcohol consumption, cigarette smoking, and risk of benign prostatic hyperplasia. Am J Epidemiol 1999;149(2):106-115.

27. Roberts RO, Jacobsen SJ, Rhodes T, Guess HA, Girman CJ, Panser LA, Chute CG, Oesterling JE, Lieber MM. Cigarette smoking and prostatism: a biphasic association? Urology 1994; 43(6):797-801.

28. Roberts RO, Tsukamoto T, Kumamoto Y, Rhodes T, Masumori N, Miyake H, Girman CJ, Jacobsen SJ, Lieber MM.. Association between cigarette smoking and prostatism in a Japanese community. Prostate 1997;30:((3):154-159.

29. Seitter WR, Barret-Connor E. Cigarette smoking, obesity, ang benign prostatic hyperthrophy: a prostective population-based study. Am J Epidemiol 1992;135(5):500-503.

30. Koskimaki J, Hakama M, Huhtala H, Tammela TL.Association of dietary elements and lower urinary tract symptoms. Scand J Urol Nephrol 2000;34(1):46-50.

31. Jankevicius F, Miller SM, Ackermann R. Nutrition and risk of prostate cancer. Urol Int 2002;68(2):69-80.

32. Nieto-García FJ, Bush TL, Kelly PM. Body mass definition of obesity, sensitivity and specificity using self-reported weight and height. Epidemiology 1990;1(2):146-152.

33. Dahle SE, Chokkalingam AP, Gao YT, Deng J, Stanczyk FZ, Hsing AW. Body size and serum levels of insulin and leptin in relation to benign prostatic hyperplasia. J Urology 2002;168 (2):599-604.

34. Lee E, Yoo KY, Kim Y, Shin y, Lee C.Prevalence of lower urinary tract symptoms in Korean men in a community-based study. Eur Urol 1998;33(1):17-21.

Correspondencia autor: Dra. Cristina Fernández Pérez Servicio de Medicina Preventiva. Hospital Clínico San Carlos Profesor Martín Lagos, s/n. - 28040 Madrid.

Tel.: 913303194

E-mail autor: cfernandez.hcsc@salud.madrid.org

Información artículo: Original - HBP

Trabajo recibido: septiembre 2008

Trabajo aceptado: octubre 2008 